\title{
Evaluation of Gut Microbial Changes in Human and Murine Models in Response to Antibiotics
}

\author{
S. KUMAR*, N. PERUMAL ${ }^{1}$ AND V. S. RAJ ${ }^{2}$
}

National Institute of Biologicals, Ministry of Health and Family Welfare, Noida 201303, Uttar Pradesh, ${ }^{1}$ National Institute of Immunology, New Delhi 110067, ${ }^{2}$ Center for Drug, Design, Discovery and Development (C4D), SRM University, Sonepat, Haryana 131023, India

\section{Kumar et al.: Gut Microbial Changes in Response to Antibiotics}

\begin{abstract}
Widespread usage of antibiotics disrupted the host bacterial mutualism in the human intestine. The antibiotic alterations can drive the functionality of the gut microbiota towards a state similar to those observed under various disease states in humans. The emergence of antibiotic-resistant bacteria further spurred the development of the antimicrobial crisis all over the world. Antibiotics have an essential role in treating various diseases. To understand the intricate relationship between antibiotics and human gut microbiota, the basic understanding of the microbial signature of gut dysbiosis in human patients and murine models in response to antibiotics treatment is very crucial. Therefore, we examined the effects of most commonly used antibiotics on human and murine gut microbiota, when administered alone or in a combination, under this article.
\end{abstract}

Key words: Antibiotics, gut ecology, murine microbiota, human microbiome

Emerging priorities towards gut microbiota research have been acknowledged across the world due to their extensive involvement in health and development or progression of diseases ${ }^{[1]}$. The human gut microbiota is a highly complex and dynamic community dominated by bacterial species belonging to Bacteroidetes, Firmicutes, Actinobacteria, Proteobacteria. Fusobacteria and Verrucomicrobia with two major phyla Firmicutes and Bacteroidetes account for $90 \%$ of gut microbiota population ${ }^{[2]}$. Prominently, the human gut ecosystem is not the static element and primarily driven by various factors like host physiology, diet, antibiotics and the interactions between individual microbes $^{[3]}$. The antibiotics are considered as magic bullets owing to their revolutionized influence in the

*Address for correspondence

E-mail: drsureshkumarmohil@gmail.com

November-December 2021 treatment of infectious disease ${ }^{[4]}$. They must apply in life-threatening conditions like sepsis or a severe lung infection. But in general, they have a devastating effect on gut microbiota and should not be taken as a casual drug. One of the most attentions is the indiscriminate, irrational and prolonged use of antibiotics that has spiked the severe microbial dysbiosis. The chaotic shift in dominating phyla, transient or profound loss of microbial diversity, a decrease of colonization resistance

This is an open access article distributed under the terms of the Creative Commons Attribution-NonCommercial-ShareAlike 3.0 License, which allows others to remix, tweak, and build upon the work non-commercially, as long as the author is credited and the new creations are licensed under the identical terms

Accepted 20 December 2021

Revised 20 February 2021

Received 30 December 2020

Indian J Pharm Sci 2021;83(6):1308-1314 
(e.g. protection against Enterobacteriaceae), overgrowth of multidrug resistant or opportunistic pathogens (e.g. Clostridium difficile), recurrence of infection and finally the development of antibiotic resistance are the main damaging consequence of severe use of antibiotics ${ }^{[5,6]}$. Several lines of evidence strengthen that microbiota have a certain degree of resilience and regain to pre-treatment state after the discontinuation of antibiotics treatment. However, the recent studies on long term effects of antibiotic intake have indicated that the emergence of Antibiotic Resistance Genes (ARGs) prevent the complete resilience in microbiota even after long periods of months and even years ${ }^{[1,7]}$. The longlasting consequences of antibiotics on human gut microbiota are generally depending upon the spectrum of factors related to host as well as antibiotics ${ }^{[7,8]}$. Various studies described the alteration of gut microbiota in human and murine models with antibiotics perturbations. Most of the studies have detailed the modulation of microbiota at the phylum level while few studies only extended the microbial shift up to the genus level. Many studies also showed the different microbiota shifts in the inter-individual ${ }^{[9]}$, inter-murine $\operatorname{model}^{[10,11]}$ and inter-species ${ }^{[12]}$ with the same antibiotic. Besides, there is a deficiency in the reporting of outcomes by different researchers on account of different techniques intended for microbial analysis. In this setting, we have endeavored to delineate the critical microbial signature of gut dysbiosis of human and murine models in response to most clinically relevant antibiotic driven perturbations. This review focuses on the impact of commonly used antibiotics either in single or in cocktails and their effects on microbiota shift in human and murine models. Effect of various classes of antibiotics on gut microbiota is discussed below. Bifidobacteria, Bacteroides thetaiotaomicron, Bacteroides fragilis, Clostridium cluster XIVa and IV are the beneficial species of gut microbiota ${ }^{[13]}$. Antibiotics are capable of changing the composition of human gut microbiota. The broad-spectrum antibiotics have a pronounced effect in diminishing microbial diversity $\left(1 / 3^{\text {rd }}\right.$ to $1 / 4^{\text {th }}$ levels $)$ as well as the exclusion of microbial niches that allow the proliferation and colonization of opportunistic pathogens like Clostridium difficile infection ${ }^{[14]}$. Several antibiotics and host-related factors have implicated in the undesirable effect of gut microbiota. Nevertheless, the accumulating scientific evidence designated that the long-term changes of gut microbiota were after treatment with well-absorbed antibiotics. An example is a cocktail of clarithromycin plus metronidazole $(4 \mathrm{y})^{[15]}$, clindamycin $(2 \mathrm{y})^{[16]}$, lincosamides $(2 \mathrm{y})^{[17]}$ ciprofloxacin $(1 \quad y)^{[18]}$ and quinolones $(1 \mathrm{y})^{[19,20]}$. The poorly absorbed antibiotics, fosfomycin, tetracycline ${ }^{[21,22]}$, sulphonamide ${ }^{[23]}$, carbapenems $^{[24,25]}$ and penicillin ${ }^{[26,27]}$ observed with short term changes on gut microbiota. Although nitrofurantoin which is well absorbed but having fast serum clearance also has minimal effect on gut microbiota $^{[23]}$. Remounting studies sustained that antibiotics treatment to humans commonly decreased the ratio of Firmicutes/Bacteroidetes due to preferential growth of Bacteroidetes over Firmicutes ${ }^{[24]}$ and the corresponding increase in the numbers of Proteobacteria $^{[25,26]}$. Actinobacteria increased with nitrofurantoin and decreased with lincosamides, macrolides, beta-lactams, fluoroquinolones, while no consistent change noticed with the aminoglycosides, tetracyclines, rifamycin, nitroimidazole and glycopeptides $^{[8,27-29]}$. Excluding nitrofurantoin, most of the antibiotic class inscribed a decrease in the relative abundance of Firmicutes ${ }^{[8,27-30]}$. The relative abundance of Bacteroidetes affirmed to increase with aminoglycosides, tetracyclines, macrolides, betalactams and fluoroquinolones groups while reduced with nitroimidazole and glycopeptides. Increase of Proteobacteria spotted in almost every class of antibiotics except in tetracycline's and lincosamides, which did not make any significant change in their abundance ${ }^{[8,27]}$. Many researchers have worked on the gut microbiota by either using the single antibiotics or in the cocktail. The treatment consequence of antibiotics at the phyla level of microbiota was almost followed similarly in human and murine species. Some evidence showed that although the qualitative differences have existed in the microbiota of humans and rodents while they have high qualitative similarity and even the faeces of both species shared the similar representatives of phyla and a large section of common genera ${ }^{[31]}$. On the contrary, few studies also informed that the inconsistency between human and animal models could be due to differences in relative dosage to administer ${ }^{[32]}$. The comparison of microbiota shift between the human and murine with the perturbations of most clinically relevant antibiotics has been provided in Table $1^{[33-38]}$ and Table $2^{[39-47]}$ and discussed further below: Antibiotic vancomycin treatment manifested similar changes in gut microbiota at the phylum level. The diminutions in the relative abundance of Firmicutes and Bacteroidetes and an increase of Proteobacteria and no relative change of Actinobacteria phyla in both human and murine species observed. However, the increase of Tenericutesin, Anaeroplasmataceae, Akkermansiaceae 
TABLE 1: EFFECT OF COMMONLY USED ANTIBIOTICS AND THEIR COMBINATIONS ON THE COMPOSITION OF HUMAN GUT MICROBIOTA

\begin{tabular}{|c|c|c|c|c|c|}
\hline Antibiotics & Actinobacteria & Firmicutes & Bacteroidetes & Proteobacteria & References \\
\hline \multirow[t]{4}{*}{ Vancomycin } & & $\uparrow$ Enterococcus & & $\uparrow$ Enterobacteriaceae & \\
\hline & & $\uparrow$ Eubacterium & & $\uparrow E$. coli & [33,34] \\
\hline & & $\downarrow$ Clostridium cluster IV \& XIVa & & $\uparrow$ Haemophilus & \\
\hline & & & & $\uparrow$ Serratia & \\
\hline \multirow{2}{*}{$\begin{array}{l}\text { Vancomycin } \\
\text { +Imipenem }\end{array}$} & & $\uparrow$ Bacillus mycoides & $\uparrow$ Tannerella & $\uparrow$ Klebsiella & \\
\hline & & $\downarrow$ Lactobacillus & $\uparrow$ Bacteroides & & \\
\hline \multirow[t]{3}{*}{ Ampicillin } & $\downarrow$ Bifidobacterium & $\uparrow$ Enterococcus & $\uparrow$ Bacteroidetes & $\uparrow$ Klebsiella & \\
\hline & & $\downarrow$ Lachnospiraceae & & $\uparrow$ Enterobacter & \\
\hline & & $\downarrow$ Lactobacillus & & & [33-35] \\
\hline \multirow{2}{*}{$\begin{array}{l}\text { Ampicillin } \\
+ \text { Gentamicin }\end{array}$} & $\downarrow$ Actinobacteria & $\downarrow$ Firmicutes & $\uparrow$ Bacteroides & $\uparrow$ Proteobacteria & \\
\hline & $\uparrow$ Bifidobacterium & $\downarrow$ Lactobacillus & & & \\
\hline \multirow[t]{4}{*}{$\begin{array}{l}\text { Ampicillin } \\
\text { +Amoxicillin }\end{array}$} & $\uparrow$ Bifidobacterium & $\uparrow$ Enterococcus & $\uparrow$ Bacteroides & $\uparrow$ Enterobactereaceae & \\
\hline & & $\uparrow$ Eubacterium & & $\uparrow$ Enterobacter & \\
\hline & & $\downarrow$ Roseburia & & $\uparrow K l e b s i e l l a$ & \\
\hline & & $\downarrow$ Veillonella & & $\downarrow$ E. coli & \\
\hline \multirow[t]{8}{*}{ Amoxicillin } & $\uparrow$ Bifidobacterium & $\uparrow$ Eubacterium & $\uparrow$ Parabacteroides & $\uparrow$ Citrobacter & \\
\hline & $\downarrow$ Collinsella & $\uparrow$ Lactobacillus & $\uparrow$ Alistipes & $\uparrow$ Enterobacter & {$[34,36]$} \\
\hline & & $\uparrow$ Ruminococcus & $\uparrow$ Bacteroides & $\uparrow K l e b s i e l l a$ & \\
\hline & & $\uparrow$ Enterococcus & & $\uparrow$ Morgenella & \\
\hline & & $\downarrow$ Coprococcus & & $\uparrow$ Shigella & \\
\hline & & $\downarrow$ Lachnospira & & $\uparrow$ Pseudomonas & \\
\hline & & $\downarrow \downarrow$ Oscillospira & & $\downarrow$ E. coli & \\
\hline & & $\downarrow \downarrow$ Roseburia & & & \\
\hline \multirow[t]{7}{*}{ Clindamycin } & $\downarrow$ Bifidobacteria & $\downarrow$ Eubacterium & $\downarrow$ Bacteroides & $\uparrow$ Citrobacter & \\
\hline & & $\downarrow$ Lactobacillus & & $\uparrow$ Enterobacter & [34] \\
\hline & & $\downarrow$ Lachnospira & & $\uparrow$ Klebsiella & \\
\hline & & $\downarrow$ Coprococcus & & $\downarrow$ E. coli & \\
\hline & & $\downarrow$ Roseburia & & & \\
\hline & & $\downarrow$ Ruminococcus & & & \\
\hline & & $\downarrow$ Streptococcus & & & \\
\hline \multirow[t]{4}{*}{ Ceftriaxone } & $\downarrow$ Bifidobacteria & $\uparrow$ Eubacterium & $\downarrow$ Bacteroides* & $\uparrow$ Klebsiella & \\
\hline & & $\uparrow$ Enterococcus & & $\downarrow$ E. coli & {$[37,38]$} \\
\hline & & $\downarrow$ Lactobacillus & & $\downarrow$ Enterobateriaceae & \\
\hline & & $\downarrow$ Clostridium & & & \\
\hline \multirow[t]{9}{*}{ Ciprofloxacin } & $\downarrow$ Bifidobacterium* & $\uparrow$ Clostridium & $\downarrow$ Parabacteroides & $\uparrow$ Citrobacter & \\
\hline & $\downarrow$ Corynebacterium & $\uparrow$ Butyricicoccus & $\downarrow$ Tannerella & $\uparrow$ Helicobacter & [34] \\
\hline & & $\uparrow$ Mediterraneibacter & $\downarrow$ Bacteroides * & $\uparrow$ Klebsiella & \\
\hline & & $\uparrow$ Blautia & & $\uparrow$ Enterobacter & \\
\hline & & $\uparrow$ Enterococcus & & $\downarrow \downarrow$ Enterobacteriaceae & \\
\hline & & $\downarrow$ Clostridium & & & \\
\hline & & $\downarrow$ Lactobacillus* & & & \\
\hline & & $\downarrow$ Veillonella & & & \\
\hline & & $\downarrow$ Ruminococcus & & & \\
\hline
\end{tabular}

Note: *Bhalodi et al. (2019) finding indicated about the stable nature of gut microbiota. $\downarrow \downarrow$-Strong suppression; $\downarrow$-Moderate suppression; $\uparrow$-Increase in number 
TABLE 2: EFFECT OF COMMONLY USED ANTIBIOTICS AND THEIR COMBINATIONS ON THE COMPOSITION OF MURINE GUT MICROBIOTA

\begin{tabular}{|c|c|c|c|c|c|}
\hline Antibiotics & Actinobacteria & Firmicutes & Bacteroidetes & Proteobacteria & References \\
\hline Vancomycin & & $\downarrow$ Lachnospiraceae & $\downarrow$ Muribaculaceae & $\uparrow$ Enterobacteriaceae & [39] \\
\hline \multirow[t]{11}{*}{ Vancomycin+Imipenem } & & $\downarrow$ Ruminococcaceae & $\downarrow$ Bacteroidaceae & $\uparrow$ Burkholderiaceae & [40] \\
\hline & & $\uparrow$ Lactobacillaceae & $\downarrow$ Prevotellaceae & $\uparrow E$. coli & \\
\hline & & $\uparrow$ Streptococcus & $\downarrow$ Rikenellaceae & $\uparrow$ Enterobacter & \\
\hline & & $\uparrow$ Lactobacillus & $\downarrow$ Bacteroidetes & $\uparrow$ Escherichia & \\
\hline & & $\uparrow$ Planomicrobium & $\downarrow \downarrow$ Odoribacter & $\uparrow$ Shigella & \\
\hline & $\downarrow \downarrow$ Enterorhabdus & $\downarrow \downarrow$ Roseburia & $\downarrow \downarrow$ Alistipes & $\uparrow$ Citrobacter & \\
\hline & & $\downarrow \downarrow$ Candidatus & $\downarrow \downarrow$ Bacteroides & $\uparrow$ Achromobacter & \\
\hline & & $\downarrow \downarrow$ Clostridium & $\downarrow \downarrow$ Parabacteroides & $\uparrow$ Salmonella & \\
\hline & & $\downarrow \downarrow$ Turicibacter & $\downarrow \downarrow R C 9$ & & \\
\hline & & $\downarrow \downarrow$ Lachnospiraceae & $\downarrow \downarrow S 24-7$ & & \\
\hline & & $\downarrow \downarrow$ Ruminococcaceae & $\downarrow \downarrow$ Prevotellaceae & & \\
\hline \multirow[t]{4}{*}{ Ampicillin } & $\downarrow$ Bifidobacterium & & $\uparrow$ Bacteroidetes & $\uparrow$ Proteobacteria & {$[41,42]$} \\
\hline & & $\begin{array}{c}\uparrow \text { Enterococcus } \\
\downarrow \text { Lachnospiraceae } \\
\downarrow \text { Coprobacillus }\end{array}$ & & $\uparrow K l e b s i e l l a$ & \\
\hline & & $\downarrow$ Dorea & & $\uparrow$ Enterobacter & \\
\hline & & $\downarrow$ Lactobacillus & & & \\
\hline Amoxicillin & $\downarrow$ Bifidobacterium & $\uparrow$ Lactobacillus & $\begin{array}{c}\uparrow \text { Bacteroidaceae } \\
\downarrow \text { Rikenellaceae }\end{array}$ & $\uparrow$ Enterobacteriaceae & \\
\hline Amoxicillin & & $\downarrow$ Clostridiales & $\downarrow$ Bacteroidetes & $\uparrow$ Proteobacteria & {$[43]$} \\
\hline \multicolumn{6}{|l|}{ Clavulanate } \\
\hline $\begin{array}{l}\text { Amoxicillin } \\
\text { +Metronidazole } \\
\text { +Bismuth }\end{array}$ & & $\downarrow$ Firmicutes & $\uparrow$ Bacteroidetes & $\begin{array}{c}\uparrow \text { Proteobacteria } \\
\uparrow \text { Enterobacteriaceae }\end{array}$ & \\
\hline Clindamycin & & & $\downarrow$ Bacteroidetes & $\begin{array}{c}\uparrow \text { Proteobacteria } \\
\uparrow E \text {. coli }\end{array}$ & [44] \\
\hline \multirow[t]{3}{*}{ Ceftriaxone } & & $\downarrow \uparrow$ Enterococcus & & & {$[45,46]$} \\
\hline & & $\uparrow$ Robinsoniella & & & \\
\hline & & $\downarrow$ Lactobacillus & & & \\
\hline \multirow[t]{5}{*}{ Ciprofloxacin } & & $\uparrow$ Coprococcus & $\uparrow$ Bacteroides & $\downarrow$ Proteobacteria & \\
\hline & & $\downarrow \downarrow$ Streptococcus & $\uparrow$ Marvinbryantia & & \\
\hline & & $\downarrow \downarrow$ Lactobacillus & $\downarrow \downarrow$ Odoribacter & & {$[40,47]$} \\
\hline & & $\downarrow \downarrow$ Clostridium & $\downarrow \downarrow$ Alistipes & & \\
\hline & & & $\downarrow$ Prevotellaceae & & \\
\hline
\end{tabular}

Note: $\downarrow \downarrow$-Strong suppression; $\downarrow$-Moderate suppression; $\uparrow$-Increase in number; $\uparrow \downarrow$ - Positive and negative effects seen in different studies

and decrease of Melainabacteria additionally perceive in murine species. The most striking that the cocktail of vancomycin plus imipenem diminished the abundance of Lactobacillus species in a human while in murine species, their increase of percentage in vancomycin treatment, as well as a cocktail of vancomycin plus imipenem detected ${ }^{[33-35,48]}$. The administration of Lactobacillus species daily to mice for $1 \mathrm{w}$ before and
$2 \mathrm{w}$ after antibiotic treatment revealed the interest finding that out of two strains of Lactobacillus, one of Lactobacillus paracasei CNCM I-3689 inoculation dramatically decreased Vancomycin-Resistant Enterococci (VRE) numbers in the faeces proclaimed an improvement of the reduction of VRE ${ }^{[48]}$. Ampicillin increased in the relative abundance of Firmicutes, Bacteroidetes and Proteobacteria in humans. Comparatively, in rodent models, the decrease of 
Firmicutes, no change in Bacteroidetes and the increase of Proteobacteria appeared. Importantly, few investigations in humans claimed the complete loss of Bacteroidetes and Verrucomicrobia. The decrease of beneficial microbes Bifidobacterium, Lachnospiraceae, Coprobacillus, Dorea and Lactobacillus in human and Bifidobacterium, Eubacterium, Subdoligranulum, Faecalibacterium and Anaerobutyricum in rodent models appeared. Apart from this, the increase of Tenericutes and Anaeroplasma has also been detected in humans. The majority of a similar trend of change of microbial shift has revealed in both species. Most interesting is that the combination of ampicillin plus gentamicin and ampicillin plus amoxicillin treatment in humans increased the Bifidobacterium ${ }^{[33-35,41,42]}$. Antibiotic, amoxicillin treatment implied the decrease in the relative abundance of Actinobacteria and Firmicutes and the increase of both Bacteroidetes and Proteobacteria in human and rodent models. The similar microbial shift is also perceived at species level including the increase of Lactobacillus, Bacteroides and Enterobacteriaceae except for Bifidobacterium that increased in humans on the contrary to rodent species. Further, the Fusobacteria was also affected in humans ${ }^{[34,36,43]}$. Clindamycin treatment made the diminution of Actinobacteria, Firmicutes and Bacteroidetes, and increased pathogenic species of Proteobacteria in humans. Bacteroidetes and Proteobacteria only declined in rodent models. The decreased in the relative abundance of Eubacterium, Lactobacillus, Lachinospira, Blautia, Coprococcus, Dorea, Veillonella, Roseburia, Ruminococcus, Streptococcus, Bacteroides, Fusobacteria and the increased of Citrobacter, Enterobacter and Klebsiella the dominant genera accounted in human. In rodent models, the contrary outcome placed in the case of Escherichia coli (E. coli) which decreased in humans but increased in rodents ${ }^{[34,4]}$. Antibiotic ceftriaxone exposure altered all dominant phyla Actinobacteria, Firmicutes, Bacteroidetes and Proteobacteria in humans while the profound changes in Firmicutes only heeded in rodent models. The reduction of Bifidobacterium, Clostridium, Lactobacillus, Bacteroides, E. coli and Enterobacteriaceae and the increase of Eubacterium, Enterococcus and Klebsiella were dominant in humans. The increase of Robinsoniella and the decrease of Enterococcus mainly reckoned in rodent models. Interestingly both, the shoot up as well as the decline of Enterococcus also cited in rodents by the researchers ${ }^{[37,38,42,45,46]}$. Ciprofloxacin treatment profoundly decreased the relative abundance of
Actinobacteria, Firmicutes and increased Proteobacteria in humans. The contrary finding of either decrease or stable for Bacteroides also acknowledged. The Firmicutes, Bacteroidetes and Proteobacteria were the main affected phyla in rodents. The decrease of Proteobacteria in contrast to the human in rodent models was the most striking conclusion. The reduction of most of the beneficial microbes Bifidobacterium, Lactobacillus, Peptostreptococcus, Veillonella, Ruminococcus, Bacillus, Parabacteroides and rise of pathobionts Citrobacter, Helicobacter, Klebsiella, Enterobacter noticed at the genus level in human. The increase of Coprococcus, Bacteroides, Marvinbryantia and decrease of Streptococcus, Lactobacillus, Clostridium, Odoribacter, Alistipes, Prevotellaceae were Turicibacter reported at the lower taxonomic level ${ }^{[34,47]}$. This review presents a concise understanding of the effect of various antibiotics on gut microbiota in both human and murine models. The antibiotic classes of nitrofurantoin increase the abundance of Actinobacteria while no significant change is observed with aminoglycosides, tetracyclines, rifamycin, nitroimidazole and glycopeptides. Most of the antibiotic classes reduce the abundance of Firmicutes except the nitrofurantoin. The glycopeptides and nitrofurantoin classes of antibiotics diminish the relative abundance of Bacteroides. Similarly, tetracycline and lincosamide reduce Proteobacteria richness. In conclusion, these antibiotics classes have the least collateral damage to gut commensal microbes. The delineation of microbiota from various studies indicates qualitative similarity in the shift of gut microbiota between the human and murine models with the perturbations of various antibiotics. However, the striking difference in the shift of selective microbial species between human and animal models has also been recognized. Therefore, the careful consideration of the effects of various antibiotics on the ecology of microbiota should be taken into account while translating the gut microbiome research results from mice models to humans. The consequences of inappropriate use of antibiotics on human health have to evoke the alarming situation across the world. The quality studies are required to understand the antibiotics turbulence within the gut microbiome for critical patients to avoid damage or replace the depleted beneficial microbiota. The use of narrow-spectrum antibiotics and the cocktail of broad-spectrum antibiotics minimize the collateral damages on gut microbiota. Currently, the use of probiotics, faecal microbiota transplantation of beneficial microbiota and phage therapy are the promising strategies to target the 
selective pathogens without disturbing the commensal gut microbiota.

\section{Conflict of interests:}

The authors declared no conflict of interest.

\section{REFERENCES}

1. Jernberg C, Löfmark S, Edlund C, Jansson JK. Long-term impacts of antibiotic exposure on the human intestinal microbiota. Microbiology 2010;156(11):3216-23.

2. Rinninella E, Raoul P, Cintoni M, Franceschi F, Miggiano GA, Gasbarrini A, et al. What is the healthy gut microbiota composition? A changing ecosystem across age, environment, diet and diseases. Microorganisms 2019;7(1):14.

3. Vrancken G, Gregory AC, Huys GR, Faust K, Raes J. Synthetic ecology of the human gut microbiota. Nat Rev Microbiol 2019;17(12):754-63.

4. Zhang Y, Limaye PB, Renaud HJ, Klaassen CD. Effect of various antibiotics on modulation of intestinal microbiota and bile acid profile in mice. Toxicol Appl Pharmacol 2014;277(2):138-45.

5. Kim S, Covington A, Pamer EG. The intestinal microbiota: Antibiotics, colonization resistance, and enteric pathogens. Immunol Rev 2017;279(1):90-105.

6. Neuman H, Forsythe P, Uzan A, Avni O, Koren O. Antibiotics in early life: Dysbiosis and the damage done. FEMS Microbiol Rev 2018;42(4):489-99.

7. De La Cochetière MF, Durand T, Lepage P, Bourreille A, Galmiche JP, Dore J. Resilience of the dominant human fecal microbiota upon short-course antibiotic challenge. J Clin Microbiol 2005;43(11):5588-92.

8. Ianiro G, Tilg H, Gasbarrini A. Antibiotics as deep modulators of gut microbiota: Between good and evil. Gut 2016;65(11):1906-15.

9. Turnbaugh PJ, Hamady M, Yatsunenko T, Cantarel BL, Duncan A, Ley RE, et al. A core gut microbiome in obese and lean twins. Nature 2009;457(7228):480-4.

10. Tulstrup MV, Christensen EG, Carvalho V, Linninge C, Ahrné S, Højberg $\mathrm{O}$, et al. Antibiotic treatment affects intestinal permeability and gut microbial composition in Wistar rats dependent on antibiotic class. PLoS One 2015;10(12):e0144854.

11. Cheng RY, Li M, Li SS, He M, Yu XH, Shi L, et al. Vancomycin and ceftriaxone can damage intestinal microbiota and affect the development of the intestinal tract and immune system to different degrees in neonatal mice. Pathog Dis 2017;75(8):1-9.

12. Krych L, Hansen CH, Hansen AK, van den Berg FW, Nielsen DS. Quantitatively different, yet qualitatively alike: A metaanalysis of the mouse core gut microbiome with a view towards the human gut microbiome. PLoS One 2013;8(5):e62578.

13. Rivière A, Selak M, Lantin D, Leroy F, De Vuyst L. Bifidobacteria and butyrate-producing colon bacteria: Importance and strategies for their stimulation in the human gut. Front Microbiol 2016;7:979.

14. Vincent C, Manges AR. Antimicrobial use, human gut microbiota and Clostridium difficile colonization and infection. Antibiotics 2015;4(3):230-53.

15. Jakobsson HE, Jernberg C, Andersson AF, Sjölund-Karlsson M, Jansson JK, Engstrand L. Short-term antibiotic treatment has differing long-term impacts on the human throat and gut microbiome. PLoS One 2010;5(3):e9836.
16. Löfmark S, Jernberg C, Jansson JK, Edlund C. Clindamycininduced enrichment and long-term persistence of resistant Bacteroides spp. and resistance genes. J Antimicrob Chemother 2006;58(6):1160-7.

17. Jernberg C, Löfmark S, Edlund C, Jansson JK. Long-term ecological impacts of antibiotic administration on the human intestinal microbiota. ISME J 2007;1(1):56-66.

18. Zaura E, Brandt BW, Teixeira de Mattos MJ, Buijs MJ, Caspers MP, Rashid MU, et al. Same exposure but two radically different responses to antibiotics: resilience of the salivary microbiome versus long-term microbial shifts in feces. mBio 2015;6(6):e01693-15.

19. Dethlefsen L, Relman DA. Incomplete recovery and individualized responses of the human distal gut microbiota to repeated antibiotic perturbation. Proc Natl Acad Sci USA 2011;108:4554-61.

20. Dethlefsen L, Huse S, Sogin ML, Relman DA. The pervasive effects of an antibiotic on the human gut microbiota, as revealed by deep 16S rRNA sequencing. PLoS Biol 2008;6(11):e280.

21. Heimdahl A, Nord CE, Weilander K. Effect of bacampicillin on human mouth, throat and colon flora. Infection 1979;7(5):S44651.

22. Rashid MU, Panagiotidis G, Bäckström T, Weintraub A, Nord CE. Ecological impact of doxycycline at low dose on normal oropharyngeal and intestinal microflora. Int $\mathrm{J}$ Antimicrob Agents 2013;41(4):352-7.

23. Mavromanolakis E, Maraki S, Samonis G, Tselentis Y, Cranidis A. Effect of norfloxacin, trimethoprim-sulfamethoxazole and nitrofurantoin on fecal flora of women with recurrent urinary tract infections. J Chemother 1997;9(3):203-7.

24. Antonopoulos DA, Huse SM, Morrison HG, Schmidt TM, Sogin ML, Young VB. Reproducible community dynamics of the gastrointestinal microbiota following antibiotic perturbation. Infect Immun 2009;77(6):2367-75.

25. Pearson H. Stomach bug makes food yield more calories. Nature 2006.

26. Brandt LJ. Fecal transplantation for the treatment of Clostridium difficile infection. Gastroenterol Hepatol 2012;8(3):191-4.

27. Gonzalez TD. Interplay between gut microbiota and antibiotics. Hauke Smidt, co-promotor, M.W.J. van Passel. Wageningen: Wageningen University; 2016.

28. Baron SA, Diene SM, Rolain JM. Human microbiomes and antibiotic resistance. Hum Microb J 2018;10:43-52.

29. Vervoort J, Xavier BB, Stewardson A, Coenen S, GodyckiCwirko M, Adriaenssens N, et al. Metagenomic analysis of the impact of nitrofurantoin treatment on the human faecal microbiota. J Antimicrob Chemother 2015;70(7):1989-92.

30. Stewardson AJ, Gaïa N, Francois P, Malhotra-Kumar S, Delemont $\mathrm{C}$, de Tejada BM, et al. Collateral damage from oral ciprofloxacin versus nitrofurantoin in outpatients with urinary tract infections: A culture-free analysis of gut microbiota. Clin Microbiol Infect 2015;21(4):344-e1.

31. Krych L, Hansen CH, Hansen AK, van den Berg FW, Nielsen DS. Quantitatively different, yet qualitatively alike: A metaanalysis of the mouse core gut microbiome with a view towards the human gut microbiome. PLoS One 2013;8(5):e62578.

32. Langdon A, Crook N, Dantas G. The effects of antibiotics on the microbiome throughout development and alternative approaches for therapeutic modulation. Genome Med 2016;8(1):1-6.

33. Khan TJ, Hasan MN, Azhar EI, Yasir M. Association of gut dysbiosis with intestinal metabolites in response to antibiotic treatment. Hum Microb J 2019;11:100054. 
34. Zimmermann P, Curtis N. The effect of antibiotics on the composition of the intestinal microbiota-a systematic review. J Infect 2019;79(6):471-89.

35. Ferrer M, Martins dos Santos VA, Ott SJ, Moya A. Gut microbiota disturbance during antibiotic therapy: A multi-omic approach. Gut Microbes 2014;5(1):64-70.

36. Fouhy F, Guinane CM, Hussey S, Wall R, Ryan CA, Dempsey $\mathrm{EM}$, et al. High-throughput sequencing reveals the incomplete, short-term recovery of infant gut microbiota following parenteral antibiotic treatment with ampicillin and gentamicin. Antimicrob Agents Chemother 2012;56(11):5811-20.

37. Knothe H, Dette GA, Shah PM. Impact of injectable cephalosporins on the gastrointestinal microflora: Observations in healthy volunteers and hospitalized patients. Infection 1985;13(1):S129-33.

38. Vogel F, Ochs HR, Wettich K, Kaiich S, Nihson-Ehle I, Odenholt I, et al. Effect of step-down therapy of ceftriaxone plus loracarbef versus parenteral therapy of ceftriaxone on the intestinal microflora in patients with community-acquired pneumonia. Clin Microbiol Infect 2001;7(7):376-9.

39. Tulstrup MV, Christensen EG, Carvalho V, Linninge C, Ahrné S, Højberg $\mathrm{O}$, et al. Antibiotic treatment affects intestinal permeability and gut microbial composition in Wistar rats dependent on antibiotic class. PLoS One 2015;10(12):e0144854.

40. Choo JM, Kanno T, Zain NM, Leong LE, Abell GC, Keeble $\mathrm{JE}$, et al. Divergent relationships between fecal microbiota and metabolome following distinct antibiotic-induced disruptions. mSphere 2017;2(1):e00005-17.
41. Shi Y, Kellingray L, Zhai Q, Gall GL, Narbad A, Zhao J, et al. Structural and functional alterations in the microbial community and immunological consequences in a mouse model of antibiotic-induced dysbiosis. Front Microbiol 2018;9:1948.

42. Roca-Saavedra P, Rodriguez JA, Lamas A, Miranda JM, Nebot C, Cardelle-Cobas A, et al. Low-dosage antibiotic intake can disturb gut microbiota in mice. CYTA J Food 2018;16(1):6728.

43. Butts CA, Paturi G, Stoklosinski H, Martell S, Hedderley D, Carpenter E. Animal model of antibiotic induced gut microbiota dysbiosis. Proceedings 2019;8(1):11.

44. Hertz FB, Budding AE, van der Lugt-Degen M, Savelkoul PH, Løbner-Olesen A, Frimodt-Møller N. Effects of antibiotics on the intestinal microbiota of mice. Antibiotics 2020;9(4):191.

45. Rosa CP, Brancaglion GA, Miyauchi-Tavares TM, Corsetti PP, de Almeida LA. Antibiotic-induced dysbiosis effects on the murine gastrointestinal tract and their systemic repercussions. Life Sci 2018;207:480-91.

46. Guo Y, Yang X, Qi Y, Wen S, Liu Y, Tang S, et al. Long-term use of ceftriaxone sodium induced changes in gut microbiota and immune system. Sci Rep 2017;7(1):1-9.

47. Boynton FD, Ericsson AC, Uchihashi M, Dunbar ML, Wilkinson JE. Doxycycline induces dysbiosis in female C57BL/6NCrl mice. BMC Res Notes 2017;10(1):1-7.

48. Nord CE, Brismar B, Kasholm-Tengve B, Tunevall G. Effect of piperacillin/tazobactam treatment on human bowel microflora. J Antimicrob Chemother 1993;31:61-5. 